\title{
Influence of time of mating and paced copulation on induction of pseudopregnancy in cyclic female rats*
}

\author{
C. A. Frye $†$ and M. S. Erskine \\ Department of Biology, Boston University, Boston, Massachusetts 02193, USA
}

\begin{abstract}
Summary. The present experiment was designed to examine whether changes occur during the course of behavioural oestrus in the sensitivity of the female to copulatory stimulation and in patterns of sexual behaviour which might influence the likelihood of luteal maintenance. Cyclic female rats were mated on the evening of pro-oestrus $(21: 00 \mathrm{~h})$ or early on the morning of oestrus $(05: 00 \mathrm{~h})$ and received either 5 or 10 intromissions from males under conditions which allowed or did not allow pacing of contacts with males to occur. During mating, the levels of sexual receptivity, the timing of sexual mounts from males, and pacing behaviours were measured. The occurrence of pseudopregnancy or pregnancy in each animal was determined by examining vaginal smears for 14 days after mating and by examining the uterus for the presence of fetuses at the end of the experiment. At both mating times, pacing of copulation with males increased the likelihood of prolonged luteal activity. However, females were more likely to become pseudopregnant following non-paced mating at 05:00 h than at 21:00 h the previous evening. Of those females receiving an ejaculation during mating, no differences were seen between groups in the incidence of pregnancy. This change in sensitivity to cervical-vaginal stimulation during oestrus was not associated with changes in sexual receptivity or pacing behaviour. The ability of cervical-vaginal stimulation to induce pseudopregnancy therefore increases toward the end of the period of oestrus, but the behavioural mechanisms which regulate receipt of such stimulation remain constant during that time.
\end{abstract}

Keywords: rat; pseudopregnancy; mating; prolactin; luteal activity

\section{Introduction}

Successful mating in the female rat requires the co-ordination of internal and external factors which are necessary to initiate the neuroendocrine changes of pregnancy. In the intact female, the cyclic release of oestrogen and progesterone from the ovaries (see Pfaff \& Schwartz-Giblin, 1988) is necessary for the expression of sexual receptivity during oestrus. At that time, sufficient cervical stimulation is received from males to trigger a neuroendocrine reflex arc inducing the twice-daily surges of prolactin which occur during the first 12 days of pregnancy (Freeman et al., 1974; Smith et al., 1976; see Gunnet \& Freeman 1983); these prolactin surges stimulate ovarian progesterone secretion from the corpora lutea which maintain pregnancy or, in the absence of fertilization, induce a 12-day period of acyclicity known as pseudopregnancy (see Gunnet \& Freeman, 1983). Thus, both the level of sexual responsiveness on the part of the female and copulatory stimulation received from males contribute to successful reproduction in this species.

Many studies have examined the particular characteristics of the copulatory stimulation required for luteal maintenance in the rat (Adler, 1969; Chester \& Zucker, 1970; Edmonds et al., 
1972; Terkel \& Sawyer, 1978; Gilman et al., 1979; Erskine et al., 1989). It is clear from the results that the frequency of vaginal intromissions received from males is a critical determinant of successful pregnancy initiation (Wilson et al., 1965; Adler, 1969; Chester \& Zucker, 1970) with a high percentage of females showing pregnancy/pseudopregnancy after receiving approximately $7-10$ intromissions. In addition, Edmonds et al. (1972) found that the interval between intromissions was also important for luteal maintenance, the optimal interval being approximately 4-15 min. Pregnancy/ pseudopregnancy could be induced in females when an interval of as much as $1 \mathrm{~h}$ elapsed between each of 10 intromissions, indicating that the intromittive stimulus is recognized and 'stored' for prolonged periods.

The behavioural mechanisms whereby the female receives appropriate cervical-vaginal stimulation for induction of pregnancy appear to involve patterns of approach toward and withdrawal from males which regulate or pace the intervals between intromissions (Bermant, 1961; Peirce \& Nuttall, 1961; Schoelch-Krieger et al., 1976; McClintock \& Anisko, 1982; Erskine et al., 1985, 1989). When experimental conditions allow the rat to pace her contacts with the male (i.e. when she is able to withdraw from the presence of the male between sexual mounts), the mean interval between intromissions is significantly longer than it is during mating tests in which the female cannot pace. In addition, the length of time she allows between each contact is a function of the intensity of the preceding stimulus received, with the contact-return time being greater after ejaculations $>$ intromissions $>$ mounts-without-intromission (Schoelch-Krieger et al., 1976; Gilman et al., 1979; Erskine, 1985). The pacing of copulatory stimulation augments the effectiveness of each intromission received, and fewer intromissions are necessary to induce pseudopregnancy under paced mating conditions compared to non-paced mating situations (Gilman et al., 1979; Erskine et al., 1989). This behavioural mechanism therefore ensures that cervical-vaginal stimulation is received at intervals optimal for maintenance of luteal function (Gilman et al., 1979; McClintock \& Anisko, 1982; Erskine et al., 1989).

While it is clear that pacing behaviours and the types of copulatory stimuli received from males are critical elements in ensuring initiation of pregnancy in the rat, it has not been determined whether pacing behaviour itself might change during the course of oestrus in the naturally cyclic female and whether these changes might be associated with an altered ability of mating stimulation to induce pregnancy/pseudopregnancy. In the experiment presented here, we chose to examine the ability of intromission frequency and pacing to maintain luteal function at two times during natural oestrus when sexual receptivity and cervical-vaginal sensitivity might be expected to differ. This study was prompted, in part, by the results of Huck et al. (1986) who demonstrated that in hamsters pregnancy rates depended upon when in the receptive period mating occurred.

\section{Materials and Methods}

Animals. Long-Evans rats $(\mathrm{N}=69$ ) were obtained from Charles River Breeding Labs (Wilmington, MA, USA) Females weighed $200-250 \mathrm{~g}$ and males weighed $300-350 \mathrm{~g}$ upon arrival. The animals were housed in like-sex cages (2$3 /$ cage) on a reversed ( $12 \mathrm{~h}$ dark: $12 \mathrm{~h}$ light) light cycle with lights on between 18:00 and 06:00 h. However, for ease of interpretation, procedures and data obtained in this experiment are presented in relation to a non-reversed light cycle (lights on 06:00-18:00 h). All rats received water and Purina Rat Chow ad libitum.

Determinations of oestrous cyclicity and sexual receptivity. Beginning 1 week after arrival, oestrous cycles were monitored by daily vaginal lavage and rats were used in an experiment only after showing 3 regular 4-day oestrous cycles. Those animals chosen for behavioural testing on a given day showed both pro-oestrous smears and lordosis responsiveness to manual palpation. The responses to three palpations were scored on a scale ranging $0-3(0=$ no lordosis to 3 = extreme dorsiflexion and head elevation; Hardy \& DeBold, 1972), and those females that showed more than one palpation score of 0 were not considered to be receptive and were not used for behavioural testing on that day.

General procedures. Paced and non-paced mating tests were carried out using previously reported procedures (Erskine, 1985). Paced mating tests occurred in a large Plexiglas chamber $(37.5 \times 75 \times 30 \mathrm{~cm})$ which was equally divided by a removable partition (Erskine, 1985). A small hole, $5 \mathrm{~cm}$ in diameter, was cut in the bottom centre of the partition to allow the female free access to the male's side of the cage. A pacing test was initiated when the receptive 
female was placed in the side of the chamber opposite the male rat. During the test the female freely entered and left the chamber containing the male. The males were unable to follow the female to the other side of the test cage because they were too big to fit through the hole; in addition, during a 5-min pretest adaptation period they had been discouraged from attempting to move through the hole by tapping them on the nose with a marking pen. During non-paced testing, the central partition was removed and the stimulus male and experimental female were allowed to mate ad lihitum. For both conditions, mating was terminated as soon as possible after the female had received the requisite number of intromissions. If an ejaculation occurred, the stimulus male was immediately replaced with a new male. No stimulus male was used more than once in any given testing session.

At 21:00 h on the evening of pro-oestrus and at 05:00 h on the morning of the day of oestrus, groups of female rats (8-10/group) were placed into the testing chamber with sexually active males and their mating behaviour was observed until they received 5 paced (5P), 10 paced (10P), 10 non-paced (10NP) or 5 non-paced (5NP) intromissions along with the mounts-without-intromission and ejaculations that occurred during the test. Following testing the animals were returned to their home cages. Their vaginal oestrous cycles were monitored daily as above for 14 days after the testing day. At autopsy the following day, the occurrence of pregnancy was determined by uterine examination.

Behavioural measurements. Behavioural events were recorded on a Tandy 100 portable computer with an interval running stopwatch. Events recorded were the occurrence of and times of entrance to and departure from the chamber containing the male, and mounts-without-intromission $(\mathrm{M})$, intromissions (I) and ejaculations (Ej). The occurrence of and intensity of lordosis in response to each copulatory mount $(M+I+E j)$ were also recorded. Behavioural measures obtained from these records were: (1) measures of the mating stimuli received, i.e. frequency of $\mathrm{M}, \mathrm{I}$, and $\mathrm{Ej}$, and the mean interval in seconds between copulatory mounts (including $\mathrm{M}, \mathrm{I}$, and Ej; inter-mount interval), intromissions ( $\mathrm{I}$ and $\mathrm{Ej}$; inter-intromission interval), and the latency in seconds to the first intromission subsequent to an ejaculation (latency to intromit after ejaculation); (2) measures of pacing behaviour, i.e. the percentage times the female left the compartment containing the male after receiving particular copulatory stimuli ( $\%$ exits after $\mathrm{M}, \mathrm{I}$ and $\mathrm{Ej})$ and latencies in seconds to return to the male compartment after these stimuli (return latency after $\mathrm{M}, \mathrm{I}$, and $\mathrm{Ej}$ ); (3) measures of sexual receptivity, i.e. the percentage occurrence of lordosis in response to mounts from the male (lordosis quotient, LQ) and mean rating of lordosis intensity (lordosis rating, LR; Hardy \& DeBold, 1972).

Statistics. The percentage of females in each group showing prolonged periods of dioestrus and pregnancy were compared using the Fisher Exact Probability test. Three way analysis of variance (ANOVA; test condition $\times$ mating time $\times$ intromission frequency; $\alpha=0.05$ ) was used for measures of mating stimuli and sexual receptivity. In addition, behaviours displayed among paced females during pacing tests were analysed using three-way ANOVAs (mating time $\times$ intromission frequency $\times$ copulatory stimulus). Post-hoc tests were Duncan's Multiple Range Tests.

\section{Results}

\section{Induction of pregnancy/pseudopregnancy}

Vaginal smear data collected throughout the 14 days after mating were examined to determine whether individual females had ceased showing oestrous cyclicity. The duration of acyclicity was variable, with animals showing between 2 and 12 consecutive days of vaginal dioestrus. The percentages of those animals in each group showing at least 12 consecutive days of dioestrous smears are presented in Fig. 1. A significant effect of pacing was seen at the $21: 00 \mathrm{~h}$ test, with $31 \cdot 6 \%$ of paced animals ( 5 and 10 intromission groups combined) and $0 \%$ of non-paced females showing prolonged dioestrous smears (Fisher Exact Probability Test: $P=0.036$ ). At the 05:00 h test, no significant overall differences were seen between paced and non-paced females, with $58.8 \%$ of paced females and $35.3 \%$ of non-paced females showing 12 or more days with dioestrous smears. However, at the $05: 00 \mathrm{~h}$ test paced females receiving 5 intromissions had a significantly greater incidence of pseudopregnancy than did non-paced females receiving 5 intromissions $(P=0.05)$. The paced and non-paced females receiving 10 intromissions at that time had a high incidence of pseudopregnancy. Since previous studies had demonstrated that cervical-vaginal stimulation of differing intensities could influence the duration of prolactin surges (with suboptimal stimulation inducing prolactin surges for less than 10 days; Gorospe \& Freeman, 1981), the data were examined to determine whether time or type of stimulation might have influenced the maintenance of acyclicity in this experiment. The data on the incidence of dioestrous smears of $\geq 6$ or $\geq 8$ days duration are presented in Table 1. The data so obtained did not differ substantially from those for the $\geq 12$ days of dioestrous smears. At 21:00 h, pacing of copulatory stimulation had an overall facilitatory effect on induction of $\geq 6(P=0.014)$ and $\geq 8(P=0.065)$ days of dioestrus, as it did for the 12-day 
measure, and there was no effect of intromission number. At 05:00 h, 5 paced intromissions increased the incidence of $\geq 8$ days of dioestrus above that seen in females receiving 5 non-paced intromissions $(P=0.02)$, while females receiving 10 paced or 10 non-paced intromissions showed equivalently high levels of dioestrus. A significant difference between the $5 \mathrm{P}$ and $5 \mathrm{NP}$ groups was not seen if 6 or more days of dioestrus was used as the measure. There was no effect of time of mating, number of intromissions, or test condition (paced $v s$ non-paced) on the incidence of pregnancy (see Table 1). However, the failure to find differences may have been due to the small number of animals receiving an ejaculation during the test in each group.

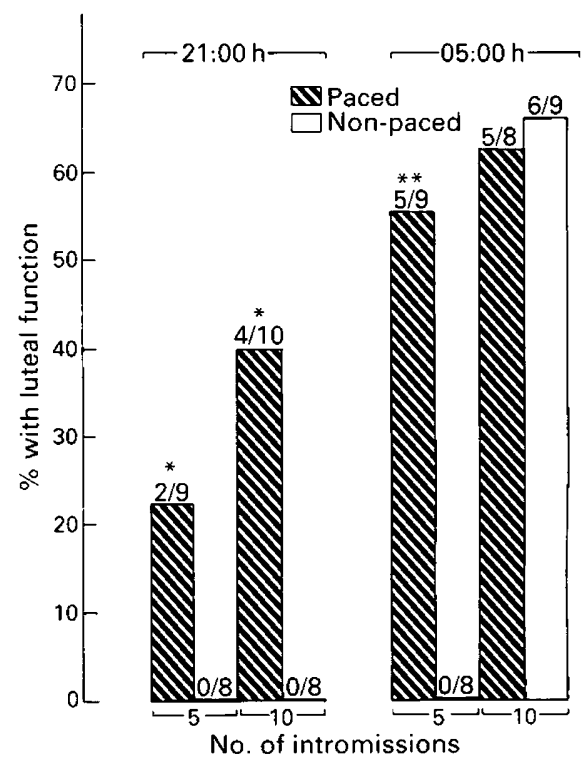

Fig. 1. Percentages of animals showing 12 consecutive days of dioestrous smears after mating with males. Groups of females received 5 or 10 intromissions in paced or non-paced mating stimulation at 21:00 $\mathrm{h}$ on the evening of pro-oestrus and 05:00 $\mathrm{h}$ on the morning of the day of vaginal oestrus. ${ }^{*}$ Significantly higher than non-paced groups at this test time, $P=0.036$. **Significantly higher than the 5 non-paced group at this time.

\section{Mating stimulation received}

Data on the frequencies of $\mathrm{M}, \mathrm{I}$, and $\mathrm{Ej}$ received by the females in each group are presented in Table 2. Overall ANOVA demonstrated that a significant difference in the frequency of $M$ occurred between females in the paced and non-paced groups $(\mathrm{F}(1,61)=8 \cdot 35, P<0.006)$, with the nonpaced groups receiving a greater number than the paced groups. A significant interaction between test condition $\times$ intromission frequency $(\mathrm{F}(1,61)=7.72, P<0.008)$ revealed that significant increases in $\mathrm{M}$ frequency occurred in the non-paced groups receiving 10 intromissions $(P<0 \cdot 05)$, while differences in $M$ frequency were not seen between the paced and non-paced groups receiving 5 intromissions. No significant differences between the paced and non-paced groups were seen in the frequency of I (an experimentally controlled variable) or Ej. However, the number of Ej received was higher overall in the groups receiving 10 intromissions than in the groups receiving 5 intromissions $(\mathrm{F}(1,61)=11.99, P<0.001)$.

Comparison of temporal characteristics of the stimulation received by the paced and non-paced females receiving 5 or 10 intromissions are presented in Fig. 2. There were no significant differences between groups tested at the two test times in the inter-mount interval, the inter-intromission 
Table 1. Percentages of animals in each group (incidence/no. tested) showing $>6$ or $>8$ days of dioestrus or becoming pregnant after receiving the mating stimulation indicated

\begin{tabular}{|c|c|c|c|}
\hline \multirow[b]{2}{*}{ Group } & \multicolumn{2}{|c|}{ Duration of dioestrus } & \multirow[b]{2}{*}{ Pregnant } \\
\hline & $>6$ days & $>8$ days & \\
\hline \multicolumn{4}{|l|}{$21: 00 \mathrm{~h}$} \\
\hline $5 P$ & $44.4(4 / 9)$ & $22 \cdot 2 \quad(2 / 9)$ & $0 \cdot 0(0 / 3)$ \\
\hline $5 \mathrm{NP}$ & $12 \cdot 5(1 / 8)$ & $12.5 \quad(1 / 8)$ & $0.0(0 / 3$ \\
\hline $10 \mathrm{P}$ & $60 \cdot 0(6 / 10)$ & $40.0 \quad(4 / 10)$ & $42 \cdot 8(3 / 7)$ \\
\hline $10 N P$ & $12 \cdot 5(1 / 10)$ & $0.0(0 / 8)$ & $0.0(0 / 3)$ \\
\hline \multicolumn{4}{|l|}{$05: 00 \mathrm{~h}$} \\
\hline $5 \mathrm{P}$ & $55.6(5 / 9)$ & $55 \cdot 6^{*}(5 / 9)$ & $100 \cdot 0(1 / 1)$ \\
\hline $5 \mathrm{NP}$ & $25 \cdot 0(2 / 8)$ & $0.0 \quad(0 / 8)$ & $0.0(0 / 3)$ \\
\hline $10 \mathrm{P}$ & $62.5(5 / 8)$ & $62 \cdot 5 \quad(5 / 8)$ & $42 \cdot 8(3 / 7)$ \\
\hline 10NP & $66 \cdot 7(6 / 9)$ & $66.7 \quad(6 / 9)$ & $57 \cdot 1(4 / 7)$ \\
\hline
\end{tabular}

Table 2. Frequency of mounts (M), intromissions (I) and ejaculations (Ej) received by cyclic females in paced and non-paced tests with males at 21:00 h on the evening of pro-oestrus and at $05: 00 \mathrm{~h}$ on day of oestrus

\begin{tabular}{|c|c|c|c|c|c|}
\hline \multirow{2}{*}{$\begin{array}{l}\text { Test } \\
\text { condition }\end{array}$} & \multirow{2}{*}{$\begin{array}{l}\text { Intromission } \\
\text { number }\end{array}$} & \multirow{2}{*}{$\begin{array}{l}\text { No. of } \\
\text { rats }\end{array}$} & \multicolumn{3}{|c|}{ Mean ( \pm s.e. $)$ frequency } \\
\hline & & & $\mathrm{M}$ & I & $\mathrm{Ej}$ \\
\hline & \multicolumn{5}{|c|}{ Pro-oestrus $21: 00 \mathrm{~h}$} \\
\hline \multirow[t]{2}{*}{ Paced } & 5 & 9 & $2 \cdot 00 \pm 0.41$ & $4.67 \pm 0.17$ & $0.33 \pm 0.17$ \\
\hline & 10 & 10 & $2.10 \pm 0.78$ & $9 \cdot 10 \pm 0.28$ & $0.80 \pm 0.20$ \\
\hline \multirow[t]{2}{*}{ Non-paced } & 5 & 8 & $0.88 \pm 0.23$ & $4 \cdot 75 \pm 0 \cdot 16$ & $0.38 \pm 0.18$ \\
\hline & 10 & 8 & $4.75 \pm 1.58^{*}$ & $9 \cdot 62 \pm 0.18$ & $0.38 \pm 0.18$ \\
\hline \multirow{3}{*}{ Paced } & \multicolumn{5}{|c|}{ Oestrus $05: 00 \mathrm{~h}$} \\
\hline & 5 & 9 & $1.22 \pm 0.43$ & $5 \cdot 11 \pm 0 \cdot 11$ & $0 \cdot 11 \pm 0 \cdot 11$ \\
\hline & 10 & 8 & $2.38 \pm 0.94$ & $9 \cdot 12 \pm 0 \cdot 12$ & $0.88 \pm 0.12$ \\
\hline \multirow[t]{2}{*}{ Non-paced } & 5 & 8 & $2.50 \pm 0.63$ & $4.62 \pm 0.18$ & $0.38 \pm 0.18$ \\
\hline & 10 & 9 & $7 \cdot 56 \pm 1.68^{*}$ & $9 \cdot 22 \pm 0.22$ & $0.78 \pm 0.15$ \\
\hline
\end{tabular}

${ }^{*} P<0.05$ compared with all other groups.

interval, and the latency to the first intromission after ejaculation, and data from both test times were combined for presentation. Inter-intromission intervals $(\mathrm{F}(1,61)=22 \cdot 68, P<0.001)$ and latencies to an intromission after ejaculation $(\mathrm{F}(1,14)=7 \cdot 28, P<0.02)$ were significantly different between paced and non-paced groups, with intervals being consistently longer for paced than for non-paced females. The inter-mount interval also was longer for the paced than for the non-paced females $(\mathrm{F}(1,45)=3.40, P<0.08)$, a difference which approached but did not achieve significance. There were no significant differences between groups receiving 5 or 10 intromissions in the intervals between $\mathrm{M}$, I, or the latency to I after Ej.

\section{Feminine sexual behaviour}

Pacing behaviour. Regulation by females of the copulatory contacts with males in the paced groups was measured by the percentage exits from the male compartment and the latency to return after different copulatory stimuli (Fig. 3). For the percentage exits measure, three-way ANOVA 

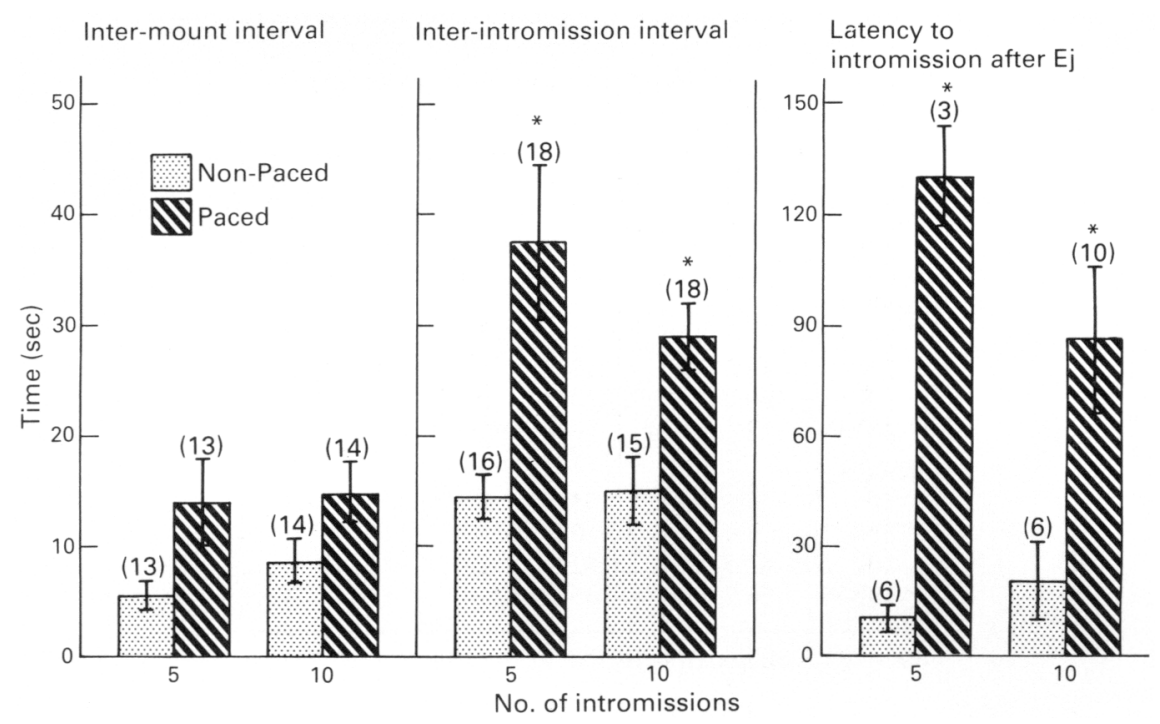

Fig. 2. Intervals between mounts and intromissions, and the latency to first intromission after ejaculation in females given 5 and 10 intromissions in paced and non-paced tests with males. Data from the two test times were combined. Values are mean \pm s.e.m. for the numbers of observations in parentheses. *Significantly higher than non-paced intervals in groups given the same numbers of intromissions, $P<0.05-0.01$.

(intromission frequency $\times$ test time $\times$ copulatory stimulus) demonstrated statistical significance for each main effect. As seen previously (Erskine, 1985; Erskine et al., 1989), the occurrence of exits after copulatory contacts increased in all groups as the intensity of the antecedent male stimulus increased $(\mathrm{M}<\mathrm{I}<\mathrm{Ej} ; \mathrm{F}(2,72)=11.23, P<0.001)$. With one exception (the single animal receiving an ejaculation in the $05: 00 \mathrm{~h} 5$ intromission group did not exit; Fig. 3a), this differential responsiveness to particular copulatory stimuli was similar regardless of time of testing or frequency of intromissions received. The two other main effects found (intromission frequency: $\mathrm{F}(1,72)=4.88, P<0.03$; and test time: $\mathrm{F}(1,72)=10.68, P<0.002)$ as well as significant interactions between test time $\times$ intromission frequency $(\mathrm{F}(1,72)=7.68, P<0.007)$ and intromission frequency $\times$ copulatory stimulus $(\mathrm{F}(2,72)=4.03, P<0.03)$ could be accounted for, again, by the lack of an exit after the single ejaculation in the $05: 00 \mathrm{~h} 5$ intromission group. We therefore conclude that there were no overall differences in percentage exits as a function of test time or intromission frequency.

Return latency data (Fig. 3b) were analysed using a three-way ANOVA as above. In many respects, these data reflect those obtained for the percentage exit measure, although there were no overall statistically significant effects of test time or intromission frequency on this measure. However, a main effect of copulatory stimulus was obtained $(\mathrm{F}(2,55)=6.05, P<0.005)$; as with the percentage exits measure, the latency to return to the male increased as a function of the intensity of the copulatory stimulus received $(\mathrm{M}<\mathrm{I}<\mathrm{Ej})$. Again, an interaction between intromission frequency $\times$ test time was seen $(\mathrm{F}(1,55)=11.24, P<0.002)$, and a three-way interaction (test time $\times$ intromission frequency $x$ copulatory stimulus, $F(2,55)=7.06, P<0.002$ ) was obtained. These latter effects were attributable to the low return latencies after $\mathrm{Ej}$ in the 05:00 $\mathrm{h} 5$ intromission group (to avoid obtaining a mean of 0 in the ANOVA, a latency of $1 \mathrm{sec}$ was assigned to that animal not showing an exit) and in the 21:00 h 10 intromission group. Return latencies after Ej in both of these groups were significantly lower $(P<0.05)$ than those recorded after Ej in the 05:00 $\mathrm{h}$ 10 intromission group and for 21:00 h 5 intromission group. 


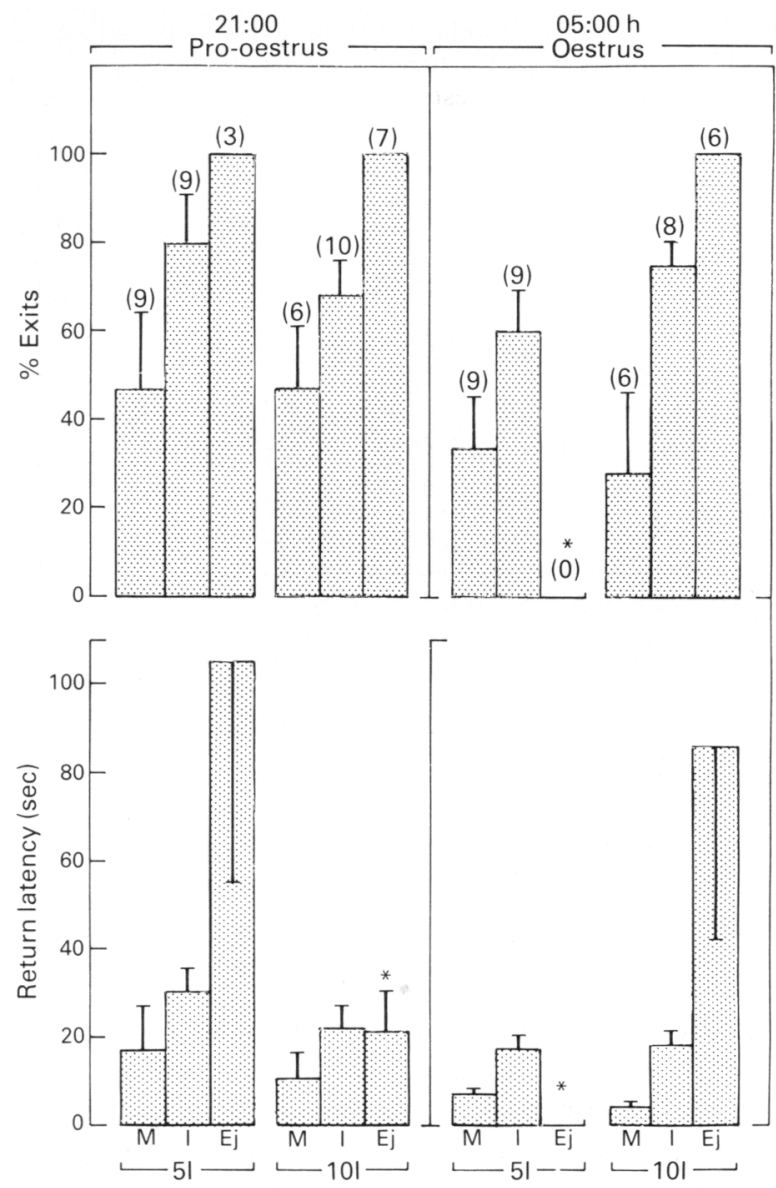

Fig. 3. Percentages of exits and return latencies exhibited during paced mating in the groups given 5 or 10 intromissions (I) at $21: 00 \mathrm{~h}$ or at $05: 00 \mathrm{~h}$. Particular antecedent male stimuli (M, I, and $\mathrm{Ej}$ ) are indicated on the abscissa. Values are mean \pm s.e.m. for the number of observations in parentheses. *Significantly lower than this measure in all other groups.

Receptivity. Lordosis quotients and lordosis ratings are presented in Table 3. A three-way ANOVA was used to assess any differences between groups on these measures (test condition $x$ test time $x$ intromission frequency). There were no statistically significant differences between or within these groups for the lordosis quotient measure, but for the lordosis rating measure there was significant effect of test time $(\mathrm{F}(1,61)=8 \cdot 14, P<0.006)$ and there was a test condition $\times$ intromission frequency interaction $(\mathrm{F}(1,61)=6.65, P<0.02)$. Over all groups, lordosis ratings were significantly elevated at the $05: 00 \mathrm{~h}$ test over $21: 00 \mathrm{~h}$ levels, with the 5 paced and $10 \mathrm{non}$-paced females showing significantly lower LRs at this time than did all other groups.

\section{Discussion}

The present data confirm, as we and others have previously reported, that pacing of copulatory stimulation by females influences the likelihood that pregnancy will occur. For groups receiving 5 or 10 intromissions, paced females were more likely than non-paced females to show 12 days of dioestrous smears if mated at $21: 00 \mathrm{~h}$ on the evening of pro-oestrus (Fig. 1; Table 1), while at 
Table 3. Lordosis quotients (LQ) and lordosis ratings (LR) exhibited by cyclic females receiving 5 or 10 intromissions and mated at $21: 00 \mathrm{~h}$ on pro-oestrus or at $05: 00 \mathrm{~h}$ on day of oestrus

\begin{tabular}{lcccc}
\hline \multirow{2}{*}{$\begin{array}{l}\text { Test } \\
\text { condition }\end{array}$} & $\begin{array}{c}\text { Intromission } \\
\text { number }\end{array}$ & $\begin{array}{c}\text { Test } \\
\text { time }\end{array}$ & \multicolumn{2}{c}{ Mean \pm s.e. } \\
\hline Paced & 5 & $21: 00 \mathrm{~h}$ & $86.7 \pm 7.7$ & LR \\
& 10 & & $99.9 \pm 0.1$ & $1.94 \pm 0.22^{*}$ \\
Non-paced & 5 & & $99.9 \pm 0.1$ & $2.38 \pm 0.18$ \\
& 10 & & $98.8 \pm 1 \cdot 2$ & $1.68 \pm 0.19^{*}$ \\
Paced & 5 & $05: 00 \mathrm{~h}$ & $95.6 \pm 2.9$ & $2.27 \pm 0.16$ \\
& 10 & & $97.5 \pm 1.6$ & $2.28 \pm 0.18$ \\
Non-paced & 5 & & $97.5 \pm 2.5$ & $2.36 \pm 0.13$ \\
& 10 & & $99.9 \pm 0.1$ & $2 \cdot 11 \pm 0.13$ \\
\hline
\end{tabular}

$* P<0.05$ compared with all other groups.

05:00 h 5 paced intromissions were significantly more likely to induce prolonged dioestrus than were 5 non-paced intromissions. Ten paced and 10 non-paced intromissions were both effective in inducing prolonged dioestrus at this later time. The particular characteristics of the stimulation received during paced mating tests that makes paced mating more effective than non-paced mating in stimulating luteal function are not known, although evidence suggests that changes in the intervals between intromissions (Gilman et al., 1979; Erskine et al., 1989; present study), the duration of each intromittive stimulus provided by the male (Erskine et al., 1989), and possibly changes in the level of the female's arousal during paced mating tests, may be responsible.

Our data suggest that the time during oestrus when mating occurs also contributes to the relative effectiveness of different types of copulatory stimulation in inducing pseudopregnancy. The facilitative effect of paced mating on induction of pregnancy/pseudopregnancy occurred at both times at which females were mated in this experiment, i.e. paced mating was overall more effective than non-paced mating. However, there appeared to be changes in the sensitivity of the females to the stimulation received at the two test times. When mating occurred during the early part of behavioural oestrus $(21: 00 \mathrm{~h})$, paced mating was more effective than non-paced mating in inducing pseudopregnancy, and there was no differential effect of intromission number; 5 and 10 intromissions induced prolonged dioestrus in approximately $30 \%$ of paced females. Females mated at 05:00 $\mathrm{h}$ also showed the enhanced pseudopregnancy response to 5 paced intromissions, but 10 paced or 10 non-paced intromissions at this time induced pseudopregnancy in a similar proportion of animals. Therefore, what had the previous evening been submaximal stimulation (10 non-paced intromissions) was sufficient to induce prolonged dioestrus at 05:00 h. These data complement those of Chester \& Zucker (1970) who reported that cervical--vaginal stimulation was more effective in inducing sperm transport in the reproductive tract late during behavioural oestrus than at earlier times. One possible explanation for the increase in prolonged dioestrus in females receiving stimulation at $05: 00 \mathrm{~h}$ is that the mating closely coincides with the timing of the daily nocturnal prolactin surge which occurs during pregnancy/pseudopregnancy (Freeman et al., 1974; Smith et al., 1976; Smith \& Neill, 1976; see Gunnet \& Freeman, 1983). The nocturnal release of prolactin is known to be dependent upon cervical-vaginal stimulation (see Gunnet \& Freeman, 1983), and increases in circulating prolactin concentration occur at the time of what would be the first nocturnal surge after artificial cervical stimulation on pro-oestrus (Smith et al., 1976). It is possible that mating stimulation at $05: 00 \mathrm{~h}$ occurs when the diurnal mechanism responsible for prolactin release is particularly susceptible to stimulation, while after mating at 21:00 h only optimal cervicalvaginal stimulation will trigger the initial release of prolactin some $8-10 \mathrm{~h}$ later. At times subsequent to $05: 00 \mathrm{~h}$ on pro-oestrus, induction of pseudopregnancy by artificial cervical-vaginal 
stimulation is again less likely (Castro-Vazquez et al., 1984). Apparently, pacing of copulatory contacts by females provides the optimal stimulation for storage of copulatory information and induction of prolactin surges when mating occurs at times before (and possibly after) the time of the nocturnal prolactin surge. An interesting possibility is that the 'storage' of the information received from cervical-vaginal stimulation which occurs after stimulation during earlier times on pro-oestrus (Smith \& Neill, 1976) may only occur if each intromission provides an easily readable signal, i.e. if the interval between each intromission is sufficiently long. The intermittent signal provided during paced mating may be a stronger signal than what might be considered to be a more continuous one received during non-paced mating, a condition found for hormonal (Parsons et al., 1982; Erskine et al., 1985), pharmacological and behavioural treatments (Post, 1980).

In light of previous findings that the intensity and timing of cervical-vaginal stimulation result in different durations of prolactin secretion (Gorospe \& Freeman, 1981), we examined our data to determine whether differences in the duration of dioestrus also occurred as a function of paced and non-paced mating stimulation (Table 1). In large part, this exercise was an unproductive one, in that similar findings were found for the $\geq 6$ and $\geq 8$ (and $\geq 10$, data not shown) dioestrous days as for the $\geq 12$ days (Fig. 1). However, electrical cervical stimulation given early on the evening of pro-oestrus failed to induce 10 or more days of prolactin surges, while addition of a second stimulus on the morning of oestrus was effective in prolonging the expression of those surges to 10 days (Gorospe \& Freeman, 1981). Although our data suggest that females are more sensitive to cervical stimulation on the morning of the day of vaginal oestrus than earlier on the previous evening, we did not see differences in the duration of dioestrus as a function of this differential sensitivity.

Although we had reported previously that the pacing of mating stimulation increased the likelihood of pregnancy as well as pseudopregnancy (Erskine et al., 1989), this possibility could not be evaluated accurately in this experiment because the number of animals receiving an ejaculation, particularly in the 5-intromission groups, was small. However, in neither study did we find any influence of paced mating or time of mating on factors other than prolongation of luteal function which might contribute to successful reproduction. Although the occurrence of 3-4 intromissions enhances sperm transport (Chester \& Zucker, 1970), numbers of implantation sites have not been shown to differ between paced and non-paced females receiving 5 intromissions (Erskine et al., 1989). The abbreviated period of behavioural oestrus which is seen following paced but not nonpaced mating (Erskine, 1985) may increase reproductive success indirectly by decreasing the time during which the behaviourally receptive female is exposed to predation.

Our results are very different from those reported for the hamster (Huck et al., 1986), in which a greater likelihood of pregnancy initiation is seen after mating early in behavioural oestrus than later. Under other conditions in which changes in sensitivity to the stimuli necessary for induction of pregnancy/pseudopregnancy have been demonstrated (i.e. age and parity), the rat and hamster appear to be similar; with advancing age and parity, increased amounts of cervical stimulation are required (Davis et al., 1977; Huck \& Lisk, 1985; Huck et al., 1988). However, the rat and the hamster differ in the types of copulatory stimuli received during mating and differ in the numbers of intromissions or ejaculatory series required for pregnancy initiation (Huck \& Lisk, 1985). For the hamster, it is not known precisely when in the circadian cycle the nocturnal prolactin surge occurs (Greenwald, 1985), and it is possible that in this species the timing of this surge coincides with the period of highest sensitivity to cervical stimulation.

In general, the quality of pacing itself was found to be very stable across the period of behavioural oestrus in rats. All four paced groups showed a clear ability to discriminate between copulatory mounts of different intensities $(\mathrm{M}, \mathrm{I}, \mathrm{Ej})$ by selectively withdrawing from the male and timing the return in response to the different stimuli. There were no overall differences in the percentage exits and return latencies (Fig. 3) or in the temporal patterning of the stimuli received (Fig. 2) as a function of test time. The one exception was the low return latencies seen in the females receiving 10 paced intromissions at 21:00 h. Since this decrease in mean return latency was not associated with a decrease in the incidence of prolonged dioestrus in this group, the normally prolonged return 
latency after ejaculation does not appear to have a significant impact on induction of pregnancy/ pseudopregnancy after paced mating.

The fairly steady level of sexual receptivity seen throughout the oestrous period and the similarity of the pacing behaviour at the two test times support the idea that pacing and lordosis behaviours are components of sexual behaviour governed by similar physiological mechanisms. However, selective changes in pacing and lordotic behaviours might have become apparent if observations had been made earlier or later in the period of behavioural oestrus. At the later test there were overall higher mean lordosis ratings than at the 21:00 h test, an effect largely the result of the significantly lower LRs in the 5P and the 10NP groups at the earlier time. However, since LQs were uniformly high for all groups and there appeared to be no systematic explanation for these lower LRs, it is reasonable to conclude that the level of sexual receptivity was for all intents and purposes maximal at both test times. The stability of both pacing and receptive behaviours demonstrates that fluctuations in sexual responsiveness do not contribute to the differential neuroendocine response of the female to cervical-vaginal stimulation at the two times.

We thank Eva Kornberg for able assistance. This research was carried out under the auspices of a grant from the Research Experiences for Undergraduate Program of the National Science Foundation. Further support was provided by HD21802 to M.S.E. from the NIH.

\section{References}

Adler, N.T. (1969) Effects of the male's copulatory behavior on successful pregnancy of the female rat. $J$. comp. Physiol. Psychol. 69, 613-622.

Bermant, G. (1961) Response latencies of female rats during sexual intercourse. Science, $N Y 133,1771-1773$.

Castro-Vazquez, A., Luque, E.H. \& Carreno, N.B. (1984) Modulation of sensitivity to cervicovaginal stimulation during the oestrous cycle: evidence for an extrapituitary action of LH-RH. Brain Research 305, 231-237.

Chester, R.V. \& Zucker, I. (1970) Influence of male copulatory behavior on sperm transport, pregnancy and pseudopregnancy in female rats. Physiol. Behav. 5, $35-43$.

Davis, H.N., Jr, Gray, G.D. \& Dewsbury, D.A. (1977) Maternal age and male behavior in relation to successful reproduction by female rats (Rattus norvegicus). $J$. comp. Physiol. Psychol. 91, 281-289.

Edmonds, S., Zoloth, S.R. \& Adler, N.T. (1972) Storage of copulatory stimulation in the female rat. Physiol. Behav. 8, 161-164.

Erskine, M.S. (1985) Effects of paced coital stimulation on estrus duration in intact cycling rats and ovariectomized and ovariectomizedadrenalectomized hormone-primed rats. J. comp. Physiol. Psychol. 99, 15I-161.

Erskine, M.S., MacLusky, N.J. \& Baum, M.J. (1985 Effect of $5 \alpha$-dihydrotestosterone on sexual receptivity and neural progestin receptors in ovariectomized rats given puised estradiol. Biol. Reprod. 33, 551-559.

Erskine, M.S., Kornberg, E. \& Cherry, J.A. (1989) Paced copulation in rats: effects of intromission frequency and duration on luteal activation and estrus length. Physiol. Behav. 45, 33--39.

Freeman, M.E., Smith, M.S., Nazian, S.J. \& Neill, J.D. (1974) Ovarian and hypothalamic control of the daily surges of prolactin secretion during pseudopregnancy in the rat. Endocrinology 94, 875-882.
Gilman, D.P., Mercer, L.F. \& Hitt, J.C. (1979) Influence of female copulatory behavior on the induction of pseudopregnancy in the female rat. Physiol. Behav. 22, 675-678.

Gorospe, W.C. \& Freeman, M.E. (1981) The effects of various methods of cervical stimulation on continuation of prolactin surges in rats. Proc. Soc exp. Biol. Med. 167, 78-82.

Greenwald, G.S. (1985) The endocrinology of the pregnant hamster. In The Hamster, pp. 53-69. Ed. H. I. Siegel. Plenum Press, New York.

Gunnet, J.W. \& Freeman, M.E. (1983) The matinginduced release of prolactin: a unique neuroendocrine response. Endocr. Rev. 4, 44-61.

Hardy, D.F. \& DeBold, J.F. (1972) Effects of coital stimulation upon behavior of the female rat. J. comp. Physiol. Psychol. 78, 400-408.

Huck, U.W. \& Lisk, R.D. (1985) Determinants of mating success in the golden hamster (Mesocricetus curatus): II. Pregnancy initiation. J. comp. Physiol. Psychol. 99, 231-239.

Huck, U.W., Lisk, R.D. \& Thierjung, C. (1986) Stimulus requirements for pregnancy initiation in the golden hamster (Mesocricetus auratus) change with time of mating during the receptive period. J. Reprod. Fert. 76, $449-458$

Huck, U.W., Lisk, R.D. \& Guyton, C.L. (1988) Stimulus requirements for pregnancy intiation in the golden hamster (Mesocricetus auratus) change with age and parity. J. Reprod. Fert. 84, 457-460.

McClintock, M.K. \& Anisko, J.J. (1982) Group mating among Norway rats $I$. Sex differences in the pattern and neuroendocrine consequences of copulation. Anim. Behav. 30, 398-409.

Parsons, B., McEwen, B.S. \& Pfaff, D.W. (1982) A discontinuous schedule of estradiol treatment is sufficient to activate progesterone-facilitated feminine sexual behavior and to increase cytosol receptors for 
progestins in the hypothalamus of the rat. Endocrinology 110, 613-619.

Peirce, J.T. \& Nuttall, R.L. (1961) Self-paced sexual behavior in the female rat. J. comp. Physiol. Psychol. 54, 310-313.

Pfaff, D.W. \& Schwartz-Giblin, S. (1988) Cellular mechanisms of female reproductive behaviors. In The Physiology of Reproduction, vol. 2, pp. 1487-1568. Eds E. Knobil, J. Neill, L. L. Ewing, G. S. Greenwald, C. L. Markert \& D. W. Pfaff. Raven Press, New York.

Post, R.M. (1980) Intermittent versus continuous stimulation: effect of time interval on the development of sensitization or tolerance. Life Sci. 26, 1275-1282.

Schoelch-Krieger, M., Orr, D. \& Perper, T. (1976) Temporal patterning of sexual behavior in the female rat. Behav. Biol. 18, 379-386.
Smith, M.S., McLean, B.K. \& Neill, J.D. (1976) Prolactin: the initial luteotropic stimulus of pseudopregnancy in the rat. Endocrinology 98, 1370-1377.

Smith, M.S. \& Neill, J.D. (1976) A "critical period" for cervically-stimulated prolactin release. Endocrinology 98, 324-328.

Terkel, J. \& Sawyer, C.H. (1978) Male copulatory behavior triggers nightly prolactin surges resulting in successful pregnancy in rats. Horm. Behav. 11, 304-309.

Wilson, J.R., Adler, N. \& LeBeouf, B. (1965) The effect of intromission frequency on successful pregnancy in the female rat. Proc. natn. Acad. Sci. USA 53, 1392-1395.

Received 29 December 1989 